\title{
RATIONAL APPROXIMATION IN THE COMPLEX PLANE AND ON THE LINE
}

\author{
TORD GANELIUS
}

\section{Introduction}

Let $K$ be a closed subset of the open set $\Omega$ in the extended complex plane and let $A=A(\Omega)$ be the set of functions holomorphic and bounded by 1 in $\Omega$. This paper is concerned with the approximation on $K$ of functions in $A$ by rational functions. In particular we are interested in finding conditions on $K, \Omega$ and $A$, which make it possible to obtain precise estimates for the degree of uniform approximation in its dependence of the number of poles of the rationals in the extended plane.

A suitable starting-point for our discussion is a beautiful result of H. Widom [22]. Let $R_{n}$ denote the set of rationals with $n$ poles and put

$$
r_{n}(f)=\inf _{g \in R_{n}} \sup _{z \in K}|f(z)-g(z)| .
$$

If $r_{n}=\sup _{f \in A} r_{n}(f)$ and if $C(K, \Omega)$ is the Green capacity of $K$ relative to $\Omega$, then under very mild restrictions on the sets involved it holds that (see $[22, \mathrm{p} .344]$ )

$$
\lim r_{n}^{1 / n}=\exp (-1 / C(K, \Omega)) .
$$

Widom gives several related results among which a lower bound for the $n$-dimensional diameter $d_{n}$ of $A$ will be of interest for us. It is defined by

$$
d_{n}=\inf _{E_{n}} \sup _{f \in A} \inf _{g \in E_{n}} \max _{z \in K}|f(z)-g(z)|,
$$

where $E_{n}$ runs through all $n$-dimensional subspaces of $\mathscr{C}(K)$. If $\Omega$ is a finite disjoint union of connected open sets with finitely many boundary components, Widom [22, Theorem 7] proves that

$$
d_{n} \geqq a \exp (-n / C(K, \Omega)) .
$$

The work reported in this paper started in July 1973 while the author was supported by the NSF-grant GP 38584 at UCSD, La Jolla, Cal., U.S.A. 
In many problems it is of considerable interest to have a more precise estimate for $r_{n}$ than the limit of $r_{n}^{1 / n}$. To obtain such estimates we certainly have to put strong restrictions on $K$ and $\Omega$. The main purpose of this paper is to present a method, which makes it possible to get precise estimates of $r_{n}$ itself - if the functions and configurations are simple enough. Rational functions of a special type will be of importance and are constructed in section 3. They are generalizations of the classical Faber polynomials so they might be called Faber rationals. The construction has certain points in common with the deduction of a formula I used in [7] without giving a full proof. The formula is closely connected with the Faber expansion (cf. Andersson [3, p. 10]) and a proof is given in section 2.

In section 4 we use the Faber rationals to obtain estimates

$$
r_{n} \leqq C_{0} \exp (-n / C(K, \Omega))
$$

in the class $A$ if the geometrical configuration is simple enough. The poles of the rationals can be chosen independently of $f$, so (1.2) shows that linear rational systems $\left\{\left(z-s_{k}\right)^{-1}\right\}_{1}^{n}$ are - within a bounded factor - as good as any other $n$-dimensional systems for approximation in $A$.

To test the precision and the applicability of the method we turn to two famous approximation problems. It is our hope that the flexibility of the method might elucidate the problem of rational approximation vs polynomial approximation. Rational approximation is better than polynomial when there is a need to distribute the poles depending on the singularities of the approximated function, and that is transparent in our method. One well-known case is Newman's [15] approximation of $|x|$ on $[-1,1]$, where there is just one singularity, and good rational approximation comes by concentration of the poles close to that singularity. Another case occurs when there is an abundance of analyticity e.g. in the case of approximation of different constants on a finite number of closed sets. If we wish to apply the previous results with $C(K, \Omega)$ we must find the best choice of $\Omega$ and it is not evident, how that is done. This problem is also discussed in section 4 and solved in a restricted case.

However, the bound obtained, does not give the precise result if it is applied to the best-known special case, i.e. the approximation of $\operatorname{sgn} x$ on two intervals $[-1, b],[b, 1], 0<b<1$, considered by Zolotarev close to hundred years ago (cf. Goncar [9]). I next show how the correct answer can be obtained by our method if $K=K_{1} \cup K_{2}$, where $K_{1}$ and $K_{2}$ are disjoint, connected closed sets and the function to approximate is constant on each of them. In a forth-coming note I shall show how to settle the case of more than two components. - In passing I give some comments on the corresponding polynomial approximation problem, for which I know the solution from a communication by W. H. J. Fuchs. 
In the last section we return to the problem of $r_{n}$ instead of $r_{n}^{1 / n}$, now in the case of piecewise holomorphic functions on intervals. The typical case is $|x|$ on $[-1,1]$, where the important step recently was taken by Vjaceslavor [18] who proved that $r_{n}(|x|) \exp (\pi \sqrt{n})$ is bounded. That is bounded from below by a positive constant has been known a few years (see Bulanov [5]). By a closer study of certain Green potentials we obtain some results including an independent proof of Vjaceslavov's result.

\section{A formula connected with the Faber expansion}

Let $K$ be a connected compact set in the complex plane, having connected complement $K^{\prime}$ and with a rectifiable boundary curve $\partial K$. We do not assume, however, that the boundary is a Jordan curve, since we want to include e.g. sets without interior like the interval. For $\zeta \in \partial K$ we define the function $v_{\zeta}$ by

$$
v_{\zeta}(s)=\arg \left(\psi\left(e^{i s}\right)-\zeta\right),
$$

where $\psi$ is the exterior mapping function. We assume that $v_{\zeta}$ is of bounded variation and that the variations have a bound independent of $\zeta$.

Let $F$ be continuous on $K$ and holomorphic in int $K$. We use the same letter $F$ to denote the harmonic extension of $F$ to $K^{\prime}$. By subtraction of a constant we may achieve that $F(\infty)=0$. With $z=x+i y$ there are two holomorphic functions $H$ and $G$ such that $F(x, y)=$ $H(z)+G(\bar{z})$ for $z \in K^{\prime}$ and $H(\infty)=G(\infty)=0$.

Take a $\zeta \in K^{\prime}$ and let $C_{\varrho}$ be a rectifiable Jordan curve surrounding $K$ and separating $\zeta$ and $K$. With the notation just introduced we find

$$
\begin{aligned}
& \int_{C_{\varrho}} F(x, y)(z-\zeta)^{-1} d z=\int_{C_{\varrho}} G(\bar{z})(z-\zeta)^{-1} d z+\int_{C_{\varrho}} H(z)(z-\zeta)^{-1} d z \\
= & -2 \pi i H(\zeta)+2 \pi i G(\bar{\zeta})+\int_{C_{\varrho}} G(\bar{z})(z-\zeta)^{-1} d z-\int_{C_{\varrho}} G(\bar{z})(\bar{z}-\bar{\zeta})^{-1} d \bar{z}
\end{aligned}
$$

and

$(2 \pi i)^{-1} \int_{C_{\varrho}} F(x, y)(z-\zeta)^{-1} d z=G(\bar{\zeta})-H(\zeta)+\frac{1}{\pi} \int_{C_{\varrho}} G(\bar{z}) d \arg (z-\zeta)$.

Our assumptions on $F$ imply that if the contour of integration on the left shrinks to $\partial K$ and if $G$ is continuous on the closure of $K^{\prime}$ (as it will be in our application), we obtain 


$$
\frac{1}{\pi} \int_{\partial K} G(\bar{z}) d \arg (z-\zeta)=H(\zeta)-G(\bar{\zeta})
$$

We next move $\zeta$ across $\partial K$ so that $\arg (z-\zeta)$ henceforward is interpreted with $\zeta$ "inside" $\partial K$. That means that we have to add $2 G(\bar{\zeta})$ to the right side to maintain the equality. Hence

$$
F(\zeta)=H(\zeta)+G(\bar{\zeta})=\frac{1}{\pi} \int_{\partial K} G(\bar{z}) d \arg (z-\zeta), \quad \zeta \in \partial K .
$$

We observe that the same formula is true if $\zeta \in \operatorname{int} K$, since by the same method

$$
\frac{1}{\pi} \int_{C_{\varrho}} G(\bar{z}) d \arg (z-\zeta)=(2 \pi i)^{-1} \int_{C_{\varrho}} F(x, y)(z-\zeta)^{-1} d z \rightarrow F(\zeta) .
$$

Formula (2.1) rewritten in the form

$$
F(\zeta)=-\frac{1}{\pi} \int_{\varrho=1}^{\infty} d \varrho \int_{s=0}^{2 \pi}(G \circ \bar{\psi})^{\prime}\left(\varrho e^{-i s}\right) e^{-i s} d v_{\zeta}(s)
$$

was applied in [7] to obtain a short proof of the following result (cf. Dzjadyk [6] and Kövari [13]).

Theorem 1. Let $K$ and $F$ be as described above. If $F \circ \psi$ satisfies a Hölder condition of order $\alpha, 0<\alpha \leqq 1$, on the unit circle, then $E_{n}(f) \leqq C n^{-\alpha}$, where $E_{n}(f)$ is the best approximation by polynomials of degree $n$.

Several complements and extensions are given in Andersson [3], where also the connection with the Faber expansion is shown. The important thing is that the Faber polynomial $P_{k}$ of order $k$ is given (except for normalization) by (cf. Pommerenke [17, Lemma 1] and [14])

$$
P_{k}(\zeta)=\int_{0}^{2 \pi} e^{i k s} d v_{\zeta}(s)=\int_{\partial K} \Phi(z)^{k} d \arg (z-\zeta),
$$

where $\Phi$ is the inverse function of $\psi$.

When we now proceed to rational approximation we shall obtain our Faber rationals by a generalization of (2.2). In [7] the proof employed Schwarz's lemma and we shall see that similarly the Blaschke factors for the poles play an important role in rational approximation. 


\section{Faber rationals}

3.1. Definition and properties. As mentioned in the introduction we shall strongly restrict the configurations we are dealing with. We shall assume that $K=\cup K_{k}$ is the union of $p$ disjoint sets, each of the type described in the previous section. The important thing is that the variation of $\arg (z-\zeta)$ is uniformly bounded when $z$ describes $\partial K$. We may e.g. assume that every $\partial K_{k}$ has bounded rotation in the sense of Paatero [16]. Sets with these properties will be said to be of type $N$.

We next prescribe the set $S_{n}$ of poles, writing $S_{n}=\left\{s_{k}\right\}_{1}^{n}$ where the points $s_{k} \in K^{\prime}$ are not necessarily distinct. The Faber rational $F\left(z ; K, S_{n}\right)$ will be a rational function of degree at most $n+p-1$. The Green function for $K^{\prime}$ singular at $s$ is denoted $g(\cdot, s)$ and a (multiplevalued) holomorphic function with real part $g(\cdot, s)$ is called $G(\cdot, s)$ so that

$$
G(\cdot, s)=g(\cdot, s)+i h(\cdot, s) .
$$

Now it is well-known (Grunsky [10, 11], ef. also Ahlfors [1] and Widom [20]) that we can (in several ways) pick $(p-1)$ points $\left\{s_{k}\right\}_{n+1}^{n+p-1}$ in $K^{\prime}$ so that

$$
\Phi_{n}(z)=\exp \left(-\sum_{1}^{n+p-1} G\left(z, s_{k}\right)\right)
$$

is a single-valued holomorphic function in $K^{\prime}$, bounded by 1 , with zeros in $S_{n}^{*}=\left\{s_{k}\right\}_{1}^{n+p-1}$ and having absolute value 1 on $\partial K^{\prime}=\partial K$.

Around every point $s_{k} \in S_{n}^{*}$ we take a negatively oriented circle $C_{k}$ and choose the radii so small that the corresponding discs $U_{k}$ are disjoint (except for coinciding $s_{k}=s_{i}$ ) and all in $K^{\prime}$. With $\zeta \in\left(\cup U_{k}\right)^{\prime}$ we define the Faber rational by

$$
\begin{aligned}
F_{n}(\zeta) & =F\left(\zeta ; K, S_{n}\right) \\
& =(2 \pi i)^{-1} \sum_{1}^{n+p-1} \int_{C_{k}} \Phi_{n}(z)^{-1}(z-\zeta)^{-1} d z+\Phi_{n}(\infty)^{-1}
\end{aligned}
$$

We collect the important properties of $F_{n}$ in

$\mathrm{Th}$ e o r e m 2. The function $F_{n}$ defined by (3.1.1) is a rational function with poles in $S_{n}^{*}$. If $K$ is of type $N$, then uniformly

$$
\begin{aligned}
& F_{n}(\zeta)=O(1), \quad \zeta \in K, \\
& F_{n}(\zeta)=\Phi_{n}(\zeta)^{-1}+O(1), \quad \zeta \in K^{\prime} .
\end{aligned}
$$

Proof. It is evident that $F_{n}$ is holomorphic in $\left(U U_{k}\right)^{\prime}$ and hence in $S_{n}^{* \prime}$. On $U_{k}$ we can write $\Phi_{n}(z)^{-1}=\left(z-s_{k}\right)^{-m} \psi(z)$ with $\psi \in H\left(U_{k}\right)$ and $\psi\left(s_{k}\right) \neq 0$. Hence 


$$
\begin{aligned}
& (2 \pi i)^{-1}\left(\zeta-s_{k}\right)^{m} \int_{C_{k}} \Phi_{n}(z)^{-1}(z-\zeta)^{-1} d z \\
& =(2 \pi i)^{-1}\left(\zeta-s_{k}\right)^{m} \int_{C_{k}} \psi(z)\left(z-s_{k}\right)^{-m}(z-\zeta)^{-1} d z \\
& =-\frac{\left(\zeta-s_{k}\right)^{m}}{(m-1) !}\left(D^{m-1} \psi(z)(z-\zeta)^{-1}\right)_{z=s_{k}} \rightarrow(-1)^{m} \psi\left(s_{k}\right)
\end{aligned}
$$

as $\zeta \rightarrow s_{k}$. We conclude that $F_{n}$ has a pole of the appropriate order at $s_{k}$.

We next move the contours from the neighbourhoods of the poles and let them tend to $\partial K$. We get one contribution from $\zeta$ if $\zeta \in K^{\prime}$ and one from infinity, the latter cancelling the last term in (3.1.1). Hence

$$
F_{n}(\zeta)=\Phi_{n}(\zeta)^{-1}+(2 \pi i)^{-1} \sum_{1}^{p} \int_{\partial K_{k}} \Phi_{n}(z)^{-1}(z-\zeta)^{-1} d z
$$

if $\zeta \in K^{\prime}$, and

$$
F_{n}(\zeta)=(2 \pi i)^{-1} \sum_{1}^{p} \int_{\partial K_{k}} \Phi_{n}(z)^{-1}(z-\zeta)^{-1} d z
$$

if $\zeta \in K$. The integrations are in the positive direction.

It is now time to use the fact that $\Phi_{n}$ has absolute value 1 on $\partial K$, so that

$$
\Phi_{n}(z)^{-1}=\overline{\Phi_{n}(z)} \quad \text { if } z \in \partial K
$$

If $\zeta \in K^{\prime}$ we get

$$
(2 \pi i)^{-1} \int_{\partial K} \overline{\Phi_{n}(z)}(\bar{z}-\bar{\zeta})^{-1} d \bar{z}=\overline{\Phi_{n}(\zeta)}+\overline{\Phi_{n}(\infty)}
$$

and thus

$$
F_{n}(\zeta)=\Phi_{n}(\zeta)^{-1}+\overline{\Phi_{n}(\zeta)}+\overline{\Phi_{n}(\infty)}+\frac{1}{\pi} \int_{\partial K} \Phi_{n}(z)^{-1} d \arg (z-\zeta)
$$

If $\zeta \in K$ we find

$$
F_{n}(\zeta)=\overline{\Phi_{n}(\infty)}+\frac{1}{\pi} \int_{\partial K} \Phi_{n}(z)^{-1} d \arg (z-\zeta)
$$

From (3.1.3) and (3.1.4) we can immediately read off the remaining statement (3.1.2) in the theorem, which hence is proved, 
3.2. Some comments on generalized Faber polynomials. It is more intricate to construct useful Faber polynomials by the method just employed. One has to choose points $\left\{t_{k}\right\}_{1}^{p-1}$ in $K^{\prime}$ so that $\exp (-n G(\cdot, \infty)+$ $\left.\sum G\left(\cdot, t_{k}\right)\right)$ is single-valued. By the methods in the literature quoted, that can be done e.g. in such a way that $\sum g\left(a, t_{k}\right)$ is minimal at a point $a \in K^{\prime}$ and the minimal value has bounds independent of the periods, i.e. independent of $n$. The polynomials are defined by

$$
P_{n}(\zeta)=(2 \pi i)^{-1} \int_{C} \Phi_{n}(z)^{-1}(z-\zeta)^{-1} d z
$$

where $C$ is a large circle in the positive direction and

$$
\Phi_{n}(z)=\exp \left(-n G(z, \infty)+\sum G\left(z, t_{k}\right)\right) .
$$

As before we get

$$
P_{n}(\zeta)=\Phi_{n}(\zeta)^{-1}+(2 \pi i)^{-1} \int_{\partial K} \Phi_{n}(z)^{-1}(z-\zeta)^{-1} d z
$$

if $\zeta \in K^{\prime}$.

This formula is good enough for most purposes. The problems come when we turn to $(2 \pi i)^{-1} \int_{\partial K} \overline{\Phi_{n}(z)}(\bar{z}-\bar{\zeta})^{-1} d \bar{z}$, with $\zeta \in K$, since we get contributions from the poles $t_{k}$. With $\zeta \in K$ we find that

$$
P_{n}(\zeta)=\frac{1}{\pi} \int_{\partial K} \Phi_{n}(z)^{-1} d \arg (z-\zeta)-\sum_{1}^{p-1} a_{k}\left(\bar{\zeta}-\bar{t}_{k}\right)^{-1},
$$

where $a_{k}$ is the residue of $\bar{\Phi}_{n}(\bar{z})$ at $\bar{z}=\bar{t}_{k}$. To get the boundedness on $K$ we have to know that the $t_{k}$ :s stay away from $\partial K$. That is not generally true with the choice above but the doubly connected case is easy to handle. Grunsky [11] gives further information about the possible choices of $\left\{t_{k}\right\}$.

\section{The approximation method}

4.1. Let us consider the problem to approximate $f \in A(\Omega)$ by rationals on a set $K$ of type $N$ in $\Omega$. It is now natural to consider compact sets in the extended plane so we assume without restriction that $\infty$ belongs to $K$. Hence $\partial \Omega$ has finite maximal distance from the origin. We take a Faber rational $F_{n}$ and consider

$$
Q_{n}(\zeta)=(2 \pi i)^{-1} F_{n}(\zeta) \int_{\Gamma} F_{n}(z)^{-1}(z-\zeta)^{-1} f(z) d z
$$


for $\zeta \in K$ and a curve (a system of curves) $T \subset \Omega$ surrounding $K$ in the positive direction. If the zeros of $F_{n}$ inside $\Gamma$ are called $\left\{a_{k}\right\}$ we have

$$
Q_{n}(\zeta)=f(\zeta)-F_{n}(\zeta) \sum F_{n}^{\prime}\left(a_{k}\right)\left(\zeta-a_{k}\right)^{-1}=f(\zeta)-G_{n}(\zeta),
$$

where $G_{n}$ is a rational function. Hence

$$
r_{n+p-1}(f) \leqq \max _{\zeta \in K}\left|Q_{n}(\zeta)\right|,
$$

and if we take $\Gamma=\partial \Omega$ we find

$$
r_{n+p-1} \leqq(2 \pi)^{-1} \max _{z \in \partial \Omega, \zeta \in K}\left|F_{n}(\zeta)(z-\zeta)^{-1}\right| \int_{\partial \Omega}\left|F_{n}(z)\right|^{-1}|d z| .
$$

The factor in front of the integral sign only depends on the geometry. We now apply 3.2. We presume that the poles are chosen so that $\min \left|\Phi_{n}(z)\right|^{-1}$ dominates the bounded term on $\partial \Omega$, and we get

$$
r_{n+p-1} \leqq C \int_{\partial \Omega}\left|\Phi_{n}(z)\right| d z \leqq C \int \exp \left(-\sum_{1}^{n} g\left(z, s_{k}\right)\right)|d z| .
$$

We now have to choose the poles. We first note that the estimate

$$
\varlimsup r_{n}^{1 / n} \leqq \exp \left(-1 / C\left(\Omega^{\prime}, K^{\prime}\right)\right)
$$

is almost immediate in our simple case. It is well-known that $C\left(\Omega^{\prime}, K^{\prime}\right)=$ $C(K, \Omega)$ (cf. Widom [22, p. 350]).

Formula (4.1.2) enables us to get more precise results. Assuming a sufficient differentiability of $\partial \Omega$ we can choose the set $S$ so that

$$
\sum g\left(z, s_{k}\right) \geqq n / C\left(\Omega^{\prime}, K^{\prime}\right)-C_{1},
$$

where $C_{1}$ depends on the geometrical configuration. When this can be done we obtain a linear set $\left\{\left(z-s_{k}\right)^{-1}\right\}$ with a degree of approximation smaller than a constant times the $n$-dimensional diameter (formula (1.2)).

I shall not pursue this matter in any generality at this place but turn to some interesting special cases. In section 5 we shall also give one example of how to take the step from continuous to discrete distribution.

4.2. The case of excessive holomorphy. A case of practical importance is the approximation by rationals of functions taking different constant values on the sets $K_{k}$. If we want to apply Widom's results or the method in 4.1 we have to choose $\Omega$ so that $C(K, \Omega)$ becomes as small as possible. In the general case that leads us to non-trivial problems connected with extremal length (where the methods in Jenkins [12] seem to be useful). 
Let us study the special case where $f$ equals 1 on $K^{*}=\mathrm{U}_{1}^{s} K_{k}$ and -1 on $K_{*}=\mathrm{U}_{s+1}^{p} K_{k}$, assuming $K=K_{*} \cup K^{*}$ to be of type $N$.

It can in fact be seen by applying the method in Goncar [9] in combination with estimates given by Widom [21, 22] that the best rational approximation $r_{n}$ satisfies

$$
\lim r_{n}^{1 / n}=\exp (-\pi d)
$$

and also $r_{n} \geqq a \exp (-\pi d n)$, where $d$ is the extremal distance of $K_{*}$ and $K^{*}$, the inverse of the capacity of the condenser formed by $K_{*}$ and $K^{*}$.

I have found it interesting to compute how small $C(K, \Omega)$ can be made in this case by a good choice of $\Omega$. It turns out that we cannot do better than

$$
C(K, \Omega)^{-1}=\frac{1}{2} \pi d
$$

The formulas for extremal length we use can be found in Ahlfors [2, Ch. 4].

We are looking for an $\Omega_{*} \supset K_{*}$ and an $\Omega^{*} \supset K^{*}$ and they shall be as large as possible so that $\Omega^{\prime}=\partial \Omega_{*}=\partial \Omega^{*}$. Let $u$ be the Green potential of the equilibrium distribution on $\partial \Omega_{*}=\partial \Omega^{*}$ and let $v$ be the harmonic function taking the values 0 on $K_{*}$ and 1 on $K^{*}$.

If $\partial \Omega_{*}=\partial \Omega^{*}$ is taken as the level line $v=1 / 2$, we have

$$
u=2 C^{-1} v \quad \text { in } \Omega_{*}, \quad u=2 C^{-1}(1-v) \quad \text { in } \Omega^{*} .
$$

With the appropriate orientation of the integration the Green formula gives

$$
\begin{aligned}
2 \pi & =C \int_{\partial \Omega_{*}} u \frac{\partial u}{\partial n} d s+C \int_{\partial \Omega^{*}} u \frac{\partial u}{\partial n} d s=C \int_{\Omega \backslash K}(\nabla u)^{2} d x d y \\
& =4 C^{-1} \iint_{\Omega}(\nabla v)^{2} d x d y
\end{aligned}
$$

where we have used the fact that $u=0$ on $\partial K$. The Dirichlet integral on the right is known to be $d^{-1}$, so we have proved that with this choice of $\Omega$ the capacity $C(K, \Omega)^{-1}=(\pi d) / 2$. If we take another boundary between $\Omega_{*}$ and $\Omega^{*}$ we can proceed as in formula $(4.2 .2)$ to obtain

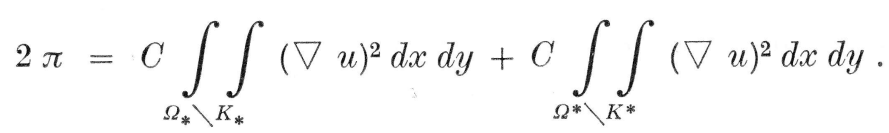

If we introduce the extremal distance $d_{*}$ from $\partial K_{*}$ to $\partial \Omega_{*}$ and $d^{*}$ from $\partial K^{*}$ to $\partial \Omega^{*}$ we get

$$
2 \pi=C^{-1}\left(d_{*_{-}-1}+d^{*-1}\right) .
$$


The harmonic mean is smaller than the arithmetic mean so it follows that

$$
C^{-1}=\pi \cdot 2\left(d_{*}^{-1}+d^{*-1}\right)^{-1} \leqq \frac{\pi}{2}\left(d_{*}+d^{* *}\right) \leqq \frac{1}{2} \pi d
$$

by the composition law. Since the level line of $v$ is smooth enough, the best approximation by rationals of our function $f$ satisfies

$$
r_{n+p-1}(f) \leqq C_{0} \exp \left(-\frac{\pi}{2} d n\right) .
$$

In 4.3 it will be shown how the special type of the function may be used to obtain the better estimate

$$
r_{n} \leqq C_{0} \exp (-\pi d n)
$$

in the case of two disjoint connected sets. The method does not generalize naturally to configurations with more sets. One way to get sharper estimates than (4.2.1) should be to introduce Faber rationals in the method applied in [9].

\subsection{Rational approximation of functions constant on two disjoint} sets. Goncar [9] proved that the best rational approximation $r_{n}$ of a function equal to +1 and -1 on two continua bounding a doubly connected domain satisfies $\lim r_{n}^{1 / n}=\left(R_{1} / R_{2}\right)^{1 / 2}$, if the domain is conformally equivalent to an annulus $\left\{w: R_{1}<|w|<R_{2}\right\}$. The special case when the continua are intervals $[-1,-b],[b, 1]$ with $0<b<1$ was already solved by Zolotarev, who found that $\left(R_{2} / R_{1}\right)^{n / 2} r_{n}$ is bounded above and below independently of $n$. I shall now state a theorem that gives a result of Zolotarev's type for a class of sets which of course is smaller than the one considered by Gončar.

The o r e m 3. Let $K_{1}$ and $K_{2}$ be disjoint sets of type $N$. If $\psi$ is a holomorphic mapping of $B=\left\{w: q^{1 / 2}<|w|<q^{-1 / 2}\right\}$ on the doubly connected domain bounded by $K_{1}$ and $K_{2}$, we further assume that to every $\omega \in \partial B$ there is a constant $p(\omega)$ so that

$$
\psi^{\prime}(w) /(\psi(w)-\psi(\omega))-p(\omega) /(w-\omega)
$$

is integrable along the boundary circles (or more generally that the condition following (4.3.8) is satisfied). Under these conditions

$$
r_{n+1} \leqq C q^{n / 2}
$$

We note that if $d$ is the extremal distance of $K_{1}$ and $K_{2}$ then $q=\exp (-2 \pi d)$. 
For the proof we define $m=[(1 / 2) n]$. As set $S^{*}$ we take $\left\{\psi\left(\varepsilon_{k}\right)\right\}_{0}^{2 m-1}$ where $\varepsilon_{k}=\exp (-i \pi k / m)$. I claim that

$$
\Phi_{n}(z)=\exp \left(-\sum_{0}^{2 m-1} G\left(z, \psi\left(\varepsilon_{k}\right)\right)\right)
$$

is single-valued and regular in $K^{\prime}=\left(K_{1} \cup K_{2}\right)^{\prime}$. That can easily be checked since $\chi_{n}=\Phi_{n} \circ \psi$ is explicitly known, the Green function for the annulus being characterized by

$$
\begin{aligned}
& \exp \left(-G\left(\psi(w), \psi\left(\varepsilon_{k}\right)\right)\right) \\
& =q^{1 / 4} v^{-1 / 2}(v-1) \prod_{s=1}^{\infty}\left(1-q^{2 s} v\right)\left(1-q^{2 s-1} v\right)^{-1}\left(v-q^{2 s}\right)\left(v-q^{2 s-1}\right)^{-1},
\end{aligned}
$$

where $v=w / \varepsilon_{k}$. Hence we get a single-valued function $\chi_{n}$ by

$$
\begin{aligned}
\chi_{n}(w) & =\Phi_{n} \circ \psi(w) \\
& =q^{m / 2} w^{-m}\left(w^{2 m}-1\right) \prod_{s=1}^{\infty} \frac{\left(1-q^{4 s m} w^{2 m}\right)\left(w^{2 m}-q^{4 s m}\right)}{\left(1-q^{2(2 s-1) m} w^{2 m}\right)\left(w^{2 m}-q^{2(2 s-1) m}\right)} .
\end{aligned}
$$

From the theory of elliptic functions we need the definition of $s n$ by

$$
\text { (4.3.3) } \operatorname{sn}(2 K x / \pi)
$$

$$
=2 q^{1 / 4} k^{-1 / 2} \sin x \prod_{s=1}^{\infty}\left(1-2 q^{2 s} \cos 2 x+q^{4 s}\right)\left(1-2 q^{2 s-1} \cos 2 x+q^{4 s}\right)^{-1},
$$

where $k$ and $K$ are well-known parameters determined by $q$ (see e.g. Whittaker - Watson [19, p. 479]), and also the Fourier series ([19, p. 510])

$$
\begin{aligned}
& \operatorname{sn}(2 K x / \pi) \\
& =2 \pi(K k)^{-1}\left\{\frac{q^{1 / 2} \sin x}{1-q}+\frac{q^{3 / 2} \sin 3 x}{1-q^{3}}+\frac{q^{5 / 2} \sin 5 x}{1-q^{5}}+\ldots\right\} .
\end{aligned}
$$

From now on $s n$ denotes the elliptic function defined by $q^{2 m}=q^{2[n / 2]}$. To stress that we write $k_{n}$ and $K_{n}$ for the corresponding quantities. With this convention (4.3.2) and (4.3.3) give

$$
\chi_{n}\left(e^{i x}\right)=k_{n}^{1 / 2} \operatorname{sn}\left(2 K_{n} m x / \pi\right) .
$$

We are now ready to apply the method of 4.1 . Let $F_{n}$ be the Faber rational corresponding to the $\Phi_{n}$ we just constructed. In formula (4.1.1) we take $\Gamma$ to be the image of $\{w:|w|=1\}$ described in one direction for $K_{1}$ and in the other for $K_{2}$. Since the function takes values with opposite signs we get

$$
\left|Q_{n}(\zeta)\right| \leqq \pi^{-1} \max _{K} F_{n} \cdot\left|\int_{I^{\prime}} F_{n}(z)^{-1}(z-\zeta)^{-1} d z\right|
$$


We now change variable by $z=\psi(w)$ and find

$$
\left|Q_{n}(\zeta)\right| \leqq C\left|\int_{|w|=1} F_{n} \circ \psi(w)^{-1}(\psi(w)-\zeta)^{-1} \psi^{\prime}(w) d w\right| .
$$

By (3.1.2) we have

$$
F_{n} \circ \psi(w)=\chi_{n}(w)^{-1}+O(1) \quad \text { on }|w|=1
$$

and (4.3.5) shows that the first term dominates, for $n$ larger than some $n_{0}$ since $s n$ is bounded by 1 . Hence

$$
F_{n} \circ \psi(w)^{-1}=\chi_{n}(w)+O(1)\left|\chi_{n}(w)\right|^{2} .
$$

Introduction in (4.3.6) gives

$$
\left|Q_{n}(\zeta)\right| \leqq C\left|\int_{|w|=1} \chi_{n}(w) \frac{w \psi^{\prime}(w)}{\psi(w)-\zeta} \frac{d w}{w}\right|+C \max \left|\chi_{n}(w)\right|^{2}
$$

It follows from our assumptions on the mapping function that the coefficients $\left(a_{s}\right)$ in the Laurent series

$$
\frac{w \psi^{\prime}(w)}{\psi(w)-\zeta}=\sum_{-\infty}^{\infty} a_{s} w^{s}
$$

satisfy $\left|a_{s}\right| \leqq C q^{|s| / 2}$. We now recall the Fourier series (4.3.4) for $\chi_{n}$ as given by (4.3.5) and insertion in the integral of (4.3.7) gives

$\left|Q_{n}(\zeta)\right| \leqq C K_{n}^{-1} k_{n}^{-1 / 2} q^{3 m / 2}\left(1-q^{2 m}\right)^{-2}+C k_{n} \leqq C q^{m}\left(1-q^{2 m}\right)^{-2} \leqq C q^{n / 2}$.

4.4. A comment on the corresponding polynomial case. The best polynomial approximation of a function equal to +1 and -1 on disjoint compact sets $K_{1}$ and $K_{2}$ was exactly determined by S. Bernstein in the case $K_{1}=[-1,-b], K_{2}=[b, 1], 0<b<1$. (An equivalent result is given on p. 120 in [4].)

Let us take a $K=K_{1} \cup K_{2}$ of type $N$ and a function $f$ coinciding on $K_{1}$ with one entire function and on $K_{2}$ with another. The Green function $g(\cdot, \infty)$ for $K^{\prime}$ singular at $\infty$ has one critical point $z_{0}$, where $g$ takes a value $w_{0}=g\left(z_{0}, \infty\right)$ such that $w_{0}$ is the smallest maximum of $g(\cdot, \infty)$ on any curve connecting $K_{1}$ and $K_{2}$. We consider the eightshaped level-line $g(z, \infty)=w_{0}$, where the two branches form an angle of $\pi / 2$ at $z_{0}$. Like in section 4.1 we consider

$$
Q_{n}(\zeta)=(2 \pi i)^{-1} P_{n}(\zeta) \int_{I^{\prime}}(z-\zeta)^{-1} P_{n}(z)^{-1} f(z) d z
$$


where now $P_{n}$ is a Faber polynomial discussed in 3.2 and $\Gamma=\Gamma_{1}+\Gamma_{2}$ consists of two curves in $\left\{z: g(z, \infty) \geqq w_{0}\right\}$, both described in the positive direction, $\Gamma_{1}$ around $K_{1}$ and $\Gamma_{2}$ around $K_{2}$, and having a line segment in common that bisects the angle of the level lines at $z_{0}$.

In a similar way as in section 4.1 we find that $\max _{\zeta \in K}\left|Q_{n}(\zeta)\right|$ is an upper bound for the best polynomial approximation $E_{n}(f)$. According to (3.2.1) the dominant contribution to the integral in (4.4.1) comes from the immediate neighbourhood of $z_{0}$. Introducing $w=G(z, \infty)$ as new variable in the integral in (4.4.1) we recall that it follows from the orthogonality mentioned above that locally $w-w_{0}=C\left(z-z_{0}\right)^{2}$ so that

$$
\left|E_{n}(f)\right| \leqq C_{0} \int_{w_{0}}^{\infty}\left(w-w_{0}\right)^{-1 / 2} \exp (-n w) d w \leqq C_{0} n^{-1 / 2} \exp \left(-n w_{0}\right) .
$$

W. H. J. Fuchs has proved that a corresponding formula gives the correct estimate for any number of sets ( $w_{0}$ being the minimal value of $g(\cdot, \infty)$ at the critical points).

If we wish to compare polynomial and rational approximation for two sets, it is relatively easy to express $w_{0}$ by elliptic functions in the symmetric case. If the doubly connected domain is conformally equivalent to an annulus with $R_{1} / R_{2}=q$, then $n^{1 / 2} k^{-n / 2} E_{n}(f)$ is bounded, to be compared with $q^{-n / 2} r_{n}(f)$ for rational approximation. Here $k$ is the modulus connected with $q$.

There is a considerable difference, since for small $k$ we have $k \sim 4 q^{1 / 2}$ and in any case $q^{1 / 2} \leqq k \leqq 4 q^{1 / 2}$. We do still worse by polynomials in the unsymmetric case while the rational approximation only depends on $q$.

\section{A remark on the best rational approximation of $|x|$}

5.1. The discussion in the previous section indicates that the good information about the poles we get by our method must be paid for by rather intricate computations. The alternative we mentioned is based on the fact that for very general sets $K_{1}$ and $K_{2}$ we have good estimates (Gončar [8], Widom [21, 22]) for

$$
\varrho_{n}=\sup _{h \in R_{n}} \max _{K_{2}}|h| / \min _{K_{1}}|h|,
$$

in particular $\varrho_{n}^{1 / n} \rightarrow \exp (-2 \pi d)$. (Cf. section 4.2.)

As far as I know the only way known to prove that we have positive upper and lower bounds for $r_{n}(|x|) \exp (\pi \sqrt{n})$ is by similar methods (Vjaceslavov [18]). Since we are dealing with the intervals $[-1,0]$ and 
$[0,1]$, which are not disjoint, we have to consider a more complicated quantity. My only aim is to show the connection with the problems discussed in 4.1. After the publication of [18] there is nothing really new in this remark, and what I should like to know is a way to carry through the computations by the methods of 4.3 .

5.2. A Green potential approximating $\log x$. Our reformulation of the crucial inequality reads as follows.

$\mathrm{T} \mathrm{h}$ e o r e m 4. Let $g(\cdot, a)$ be the Green function for the right half-plane singular at $a$. There is a positive measure $\mu$ of total mass $\omega$ on $[0,1]$, such that

$$
\int g(x, y) d \mu(y) \geqq \log x+\pi \sqrt{\omega}-C,
$$

for $x$ on $[0,1]$ and with a numerical $C$. If $\omega=n$ we can take $\mu$ as a discrete measure with integer masses.

It is rather easy to see that

$$
d v(y)=(\log y+\pi \sqrt{\omega}) y^{-1} d y \quad \text { on }[\exp (-\pi \sqrt{\omega}), 1]
$$

is a good candidate. If we put $\varphi(u)=\exp (\pi \sqrt{u})$ and take $y_{k}=\varphi(k) / \varphi(n)$, we evidently have $v\left(y_{k}, y_{k+1}\right)=1$ and an approximating discrete potential is thus given by

$$
\sum_{k=0}^{n} g\left(x, y_{k}\right)=\sum_{k=0}^{n} \log \left|\frac{x+y_{k}}{x-y_{k}}\right|=-v(x) .
$$

The inequality (5.2.1) is trivial if $0<x<\exp (-\pi \sqrt{n})$, so we suppose that $y_{k}<x \leqq y_{k+1}$ for some $k$. Fixing $x$ in this way we see that $g(x, \varphi(\cdot))$ has two convex parts; on each of them the trapezoid method gives an upper approximation. Hence with $y=\varphi(u)=\exp (\pi \sqrt{u})$ and $\xi=$ $x \exp (\pi \sqrt{n}) \geqq 1$,

$$
v(x)-C \leqq \int_{0}^{n} \log \left|\frac{\xi-\varphi(u)}{\xi+\varphi(u)}\right| d u=\frac{2}{\pi^{2}} \int_{1}^{\varphi(n)} \log \left|\frac{\xi-y}{\xi+y}\right| \log y \frac{d y}{y},
$$

the only tricky part being to show that

$$
\frac{2}{\pi^{2}} \int_{\varphi(k)}^{\varphi(k+1)} \log \left|\frac{\xi-y}{\xi+y}\right| \log y \frac{d y}{y}+\frac{1}{2} g(x, \varphi(k))+\frac{1}{2} g(x, \varphi(k+1)) \geqq-C .
$$

We observe that substitution of $\xi^{-1} \log \varphi(k)$ for $y^{-1} \log y$ in the integral only gives a bounded difference. In the resulting integral we put $y=\xi t$ and with $1+\delta=\varphi(k+1) / \xi, 1-\varepsilon=\varphi(k) / \xi$, it remains to prove that 


$$
(\delta+\varepsilon)^{-1} \int_{1-\varepsilon}^{1+\delta} \log \left|\frac{1-t}{1+t}\right| d t-\frac{1}{2} \log \frac{\delta}{2+\delta}-\frac{1}{2} \frac{\varepsilon}{1+\varepsilon} \geqq-C .
$$

Since the integral can be explicitly calculated that is completely elementary and (5.2.3) follows.

We now turn to the right member of (5.2.2). The substitution $y=\xi u$ gives

$$
\begin{gathered}
v(x)-C \leqq \frac{2}{\pi^{2}} \int_{\xi^{-1}}^{x^{-1}}(\log u+\log \xi) \log \left|\frac{1-u}{1+u}\right| \frac{d u}{u} \\
\leqq \frac{1}{2}+\frac{2}{\pi^{2}} \log \xi \int_{0}^{\infty} \log \left|\frac{1-u}{1+u}\right| \frac{d u}{u}+\frac{2}{\pi^{2}} \log \xi\left[\int_{x^{-1}}^{\infty}+\int_{0}^{\xi^{-1}} \log \left|\frac{1+u}{1-u}\right| \frac{d u}{u}\right]
\end{gathered}
$$

so that by aid of the value $-\pi^{2} / 2$ of the first integral we get

$$
v(x)+\log \xi-C \leqq \frac{2}{\pi^{2}} \log \xi\left[4 x+4 \xi^{-1}\right] \leqq C+x \pi \sqrt{n} .
$$

Since

$$
\sqrt{n} \log \left|\frac{1+x}{1-x}\right| \geqq 2 x \sqrt{n}
$$

we find that

$$
\begin{aligned}
u(x) & =\sum_{k=0}^{n} \log \left|\frac{x+y_{k}}{x-y_{k}}\right|+2[\sqrt{n}] \log \left|\frac{x+1}{x-1}\right| \\
& \geqq \log \xi-C=\log x+\pi \sqrt{n}-C .
\end{aligned}
$$

Putting $\omega=n+2[\sqrt{n}]$ and observing that $\sqrt{n} \geqq \sqrt{\omega}-1$ we have proved the estimate (5.2.1) in the theorem.

That means that we have a rational function $g$ of degree $n$ such that

$$
x|g(x)| \leqq C \exp (-\pi \sqrt{n})
$$

for $0<x \leqq 1$, and $g(-x) g(x)=1$. As in the original work [15] we immediately see that

$$
h(x)=x \frac{1-g(x)}{1+g(x)}
$$

approximates $|x|$ better than $C \exp (-\pi \sqrt{n})$. 


\section{References}

[1] Ahlfors, L. V.: Bounded analytic functions. - Duke Math. J. 14, 1947, 1- 11.

[2] -»- Conformal invariants. Topics in geometric function theory. - McGrawHill Series in Higher Mathematics. McGraw-Hill Book Company, New York etc., 1973.

[3] AndERsson, J.-E.: On the degree of polynomial and rational approximation of holomorphic functions. - Dissertation, University of Göteborg, 1975.

[4] BERNSTEIN, S.: Leçons sur les propriétés extrémales est la meilleure approximation des fonctions analytiques d'une variable réelle. - Collection de Monographies sur la Théorie des Fonctions publiée sous la direction de M. Émile Borel. Gauther-Villars et Cie, Éditeurs, Paris, 1926.

[5] Bulanov, A. Р. [А. П. Буланов]: Асимптотика для наименьщих уклонений $|x|$ от рациональьных функций. - Mat. Sb. (N.S.) 76 (118), 1968, 288303. Translation: Asymptotics for least deviation of $|x|$ from rational functions. - Math. USSR-Sb. 5, 1968, 275-290.

[6] DzJadyK, V. K. [В. К. Дзядык]: Исследования по теории приближений аналитических функйий, проводимые в Институте математики АН УССР. [Investigations in the theory of approximations of analytic functions conducted at the Institute of Mathematics of the Ukrainian Academy of Sciences.] - Ukrain. Mat. Ž. 21, 1969, 173-192.

[7] Ganelius, T. H.: Degree of approximation by polynomials on compact plane sets. - Approximation theory [Proceedings, International symposium, Austin, Texas, 1973, edited by G. G. Lorentz], Academic Press, Inc., New York-London, 1973, 347-351.

[8] Gončar, A. A. [A. A. Гончар]: Об обобщенном аналитическом продолжении. Mat. Sb. (N.S.) 76 (118), 1968, 135-146. Translation: On a generalized analytic continuation. - Math. USSR-Sb. 5, 1968, 129-140.

[9] -»- О задачах Е. И. Золотарева, связанных с рациональными функциями. - Mat. Sb.(N.S.) 78 (120), 1969, 640-654. Translation: Zolotarev problems connected with rational functions. - Math. USSR-Sb. 7, $1969,623-635$.

[10] Grunsky, H.: Eindeutige beschränkte Funktionen in mehrfach zusammenhängenden Gebieten. I. - Jber. Deutsch. Math.-Verein. 50, 1940, $230-255$.

[11] -»- Eindeutige beschränkte Funktionen in mehrfach zusammenhängenden Gebieten. II. - Jber. Deutsch. Math.-Verein. 52, 1942, 118-132.

[12] JEnkins, J. A.: Symmetrization results for some conformal invariants. - Amer. J. Math. 75, 1953, 510-522.

[13] KövarI, T.: On the order of polynomial approximation for closed Jordan domains. - J. Approximation Theory 5, 1972, 362-373.

[14] Kövari, T., and CH. Pommerenke: On Faber polynomials and Faber expansions. - Math. Z. 99, 1967, 193-206.

[15] Newman, D. J.: Rational approximation to $|x|$. - Michigan Math. J. 11, 1964, 11-14.

[16] PaATERo, V.: Über die konforme Abbildung von Gebieten deren Ränder von beschränkter Drehung sind. - Ann. Acad. Sci. Fenn. Ser. A. 33:9, 1931, $1-79$.

[17] Pommerenke, Ch.: Konforme Abbildung und Fekete-Punkte. - Math. Z. $89,1965,422-438$. 
[18] VJaČESLAVOV, N. S. [H. C. Вячеславов]: О равномерном приближении $|x|$ рациональными функциями. - Dokl. Akad. Nauk SSSR 220, 1975, 512-515. Translation: On uniform approximation of $|x|$ by rational functions. Soviet Math. Dokl. 16, 1975, 100-104.

[19] Whit' edition.] University Press, Cambridge, 1927.

[20] Widom, H.: Extremal polynomials associated with a system of curves in the complex plane. - Advances in Math. 3, 1969, 127-232.

[21] -»- An inequality for rational functions. - Proc. Amer. Math. Soc. 24, $1970,415-416$.

[22] -»- Rational approximation and n-dimensional diameter. - J. Approximation Theory 5, 1972, 343-361.

\section{University of Göteborg}

Department of Mathematics

S-402 20 Göteborg 5

Sweden

Received 2 September 1975 